\title{
Focus on gastrointestinal bleeding
}

\author{
Y. A. Kılıç
}

Published online: 14 July 2011

(c) Springer-Verlag 2011

Gastrointestinal bleeding is a common life-threatening problem, causing significant mortality, costs and resource allocation. Appropriate management of gastrointestinal bleeding requires a dynamic multidisciplinary approach in which surgeons, gastroenterologists, radiologists, intensivists and nuclear medicine specialists are involved. This multidisciplinary process must be well orchestrated; priorities in diagnostic and therapeutic interventions should be decided upon and made clear, and initial management and resuscitation should be well coordinated.

The main objective of the management of gastrointestinal bleeding is to identify and control the source of bleeding while restoring and maintaining vital organ perfusion. This approach should aim to minimize transfusion requirements by early control of the source of bleeding using the therapeutic intervention that is associated with the lowest rates of complications and rebleeding.

Lessons learned from studies in trauma patients resulted in important advances in the management of hemorrhagic shock. However, these advances in resuscitation and monitoring have not yet been fully adapted to the management of gastrointestinal bleeding [1]. Resuscitation of severe gastrointestinal bleeding requires a delicate balance that maintains appropriate end organ perfusion to prevent organ failure while avoiding the complications of aggressive resuscitation which may increase hemorrhage and mortality in uncontrolled hemorrhage. In order to delicately tailor resuscitation to patient's needs, one must use dynamic predictors of volume responsiveness within the

Y. A. Kılıç $(\bowtie)$

Department of General Surgery, Faculty of Medicine,

Hacettepe University, 06100 Ankara, Turkey

e-mail: yusufa@hacettepe.edu.tr concept of functional hemodynamic monitoring. The transfusion decision must be based on good clinical judgement and physiologic transfusion triggers. Blood products must be used within the principles of hemostatic resuscitation, with the early use of fresh frozen plasma and platelet transfusions in a 1:1:1 ratio. The management strategy should be to prevent rather then to wait for development of coagulopathy and thrombocytopenia. Transfusion-associated acute lung injury is a major cause of morbidity in patients with gastrointestinal bleeding, and a more restrictive plasma transfusion approach may be appropriate in these patients, especially those with endstage liver disease.

Advances in endoscopic and radiologic procedures have changed the priorities in the management of gastrointestinal bleeding [2]. On the other hand, prolonged attempts at the localization and control of the bleeding site increases transfusion requirements and mortality, and a timely decision to operate may be life-saving. Endoscopy is the preferred initial diagnostic and therapeutic intervention in actively bleeding patients, but local expertise and the availability of resources may direct the decision of the surgeon. The surgeon, as the person who will be in the position to orchestrate the resuscitation and treatment of the patient, must be familiar with the advantages and drawbacks of alternative diagnostic and therapeutic methods. The episodic nature of the bleeding and risk of rebleeding must be considered for decision-making.

Today, computed tomographic angiography is considered the preferred diagnostic method for cases in which emergency endoscopy has failed. Due to advances in spatial and time resolution, the use of three-dimensional maximum intensity projections and multiplanar reconstructions, and the ability to detect bleeding as slow as $0.3 \mathrm{ml} / \mathrm{min}$, CT angiography is more sensitive than 
angiography for localizing the bleeding site [3]. Additionally, CT can provide additional clues regarding the etiology of bleeding. Bowel ischemia has become a lesser concern due to the use of superselective targeted embolization with coaxial microcatheters.

One of the most challenging scenarios is to operate on a patient with major lower gastrointestinal bleeding in whom the preoperative identification of the bleeding source was not possible. Blind segmental resections are associated with a rebleeding rate of $47 \%$ and a mortality rate of $57 \%$, and should be avoided. If the patient's hemodynamic status and comorbidities and the availability of resources allow it, intraoperative endoscopy can aid in localization and allow a directed resection in selected cases. Otherwise, subtotal colectomy must be preferred. The small bowel is an important source of lower gastrointestinal bleeding, and a bleeding site in the small bowel must be considered before making the decision to perform colectomy. Fortunately, advances in endoscopic and radiologic techniques have improved the success rates for identifying and controlling the source of bleeding in these patients [4].
The papers published in this issue represent the multidisciplinary approach to management of gastrointestinal bleeding, and we hope they will be interesting and useful to readers.

Conflict of interest None.

\section{References}

1. Kılıç YA, Konan A, Kaynaroğlu V. Resuscitation and monitoring in gastrointestinal bleeding. Eur $\mathrm{J}$ Trauma Emerg Surg. 2011;37(4). doi:10.1007/s00068-011-0113-6.

2. Jovanovic I, Vormbrock K, Wilcox C, Mönkemüller K. Therapeutic and interventional endoscopy for gastrointestinal bleeding. Eur J Trauma Emerg Surg. 2011;37(4). doi:10.1007/s00068011-0125-2.

3. Friebe B, Wieners G. Radiographic techniques for localization and treatment of gastrointestinal bleeding of obscure origin. Eur $\mathrm{J}$ Trauma Emerg Surg. 2011;37(4). doi:10.1007/s00068-011-0128-z.

4. Pfeifer J. Surgical management of lower gastrointestinal bleeding. Eur J Trauma Emerg Surg. 2011;37(4). doi:10.1007/s00068011-0122-5. 